\title{
Improving Dental School Clinic Operations Using Business Intelligence Data
}

\author{
Romesh P. Nalliah, Kelly Miller, Stephen Stefanac
}

Abstract: In dental school clinics, students spend a great deal of time waiting for faculty members to check and approve their work. Traditionally at the University of Michigan School of Dentistry, students have left their cubicles to form lines behind supervising faculty members, and this line of students would follow the faculty member around from patient to patient. To address this problem and improve the patient experience, the school computerized the approval-seeking process by building the Faculty Request System (FRS) to enable students to stay with their patients while seeking the necessary approvals. The FRS produced a large volume of time-stamped, business intelligence data that enabled further evaluation. The aim of this study was to assess the effects of this change, including the quality/process improvement interventions that were possible due to information revealed by the FRS. The results showed no change in the number of students or faculty members per clinic session across the three years of this evaluation. With the FRS, the amount of time students spent away from their patients was reduced from 40.6 minutes to 12.1 minutes. After the FRS was implemented, there was an eradication of appointments that ended 30 minutes late (from $0.03 \%$ to zero) and a reduction of appointments that ended 15 minutes late (from $0.25 \%$ to $0.01 \%$ ). There were also increases in students' starting appointments on time ( $9.8 \%$ of start checks to $25.8 \%$ ), 15 minutes late ( $16.6 \%$ to $35.2 \%$ ), and 30 minutes late ( $13.2 \%$ to $22.2 \%$ ). By critically analyzing data from the new system, the school's leadership can analyze trends and make data-driven decisions to alter operations. The results of this study suggest that this process can improve the patient and student experience and faculty utilization.

Romesh P. Nalliah, DDS, MHCH, is Director of Clinical Education in DDS Program, University of Michigan School of Dentistry; Kelly Miller, BS, is Senior Business Intelligence Analyst, Michigan Medicine, Ann Arbor, MI; and Stephen Stefanac, DDS, MS, is Senior Associate Dean, University of Michigan School of Dentistry. Direct correspondence to Dr. Romesh P. Nalliah, University of Michigan School of Dentistry, 1011 N. University, Ann Arbor, MI 48109; romeshn@umich.edu.

Keywords: dental education, dental school clinic, patient safety, patient satisfaction, information management, computer applications, clinic management

Submitted for publication 8/6/18; accepted 12/4/18; first published online 3/25/19 doi: 10.21815/JDE.019.080

$\mathrm{D}$ ental school clinics are unique environments in which to receive dental services since care is delivered by novice providers under the supervision of licensed dentists. Patients must understand that care is slower, multiple treatment opinions will be sought, and treatment must be occasionally redone to ensure high quality. Each step that students perform during a dental procedure must be checked and approved by a faculty member before moving on to the next step. As a consequence, much time is spent waiting for faculty members, who are managing multiple cases at the same time.

A study at the University of Kentucky School of Dentistry evaluated patient satisfaction when appointment length in the emergency clinic was reduced from four hours to three hours using Lean processes. ${ }^{1}$ Reducing the length of the appointment may also enable care to be delivered to more patients; however, that factor was not measured or reported in that study.
A study at Harvard School of Dental Medicine found that students on externship rotation at a community health center billed double the number of treatment codes than when they were in the highly controlled dental school clinic environment. ${ }^{2}$ Externships can allow supervising dentists at a community rotation to form a closer relationship with the students and entrust them with more responsibility and fewer step-checks, which may increase productivity. This model is replicated in medical residencies, and there has been early interest in bringing entrustable professional activities (EPAs) to dental schools. ${ }^{3}$ However, it should be noted that revenues generated in the Harvard study were equivalent whether students were in the dental school clinic or community rotation, so the authors concluded that students were doing more complex (and more expensive) procedures in the school clinic but simpler (and less expensive) procedures in high volume on the community rotation. 
Recent studies have reported a large amount of variation between dental schools in hours students spend in clinical care, procedural experience, and revenue generation. ${ }^{4,5}$ This difference may mean that improving efficiency could have very different meanings and pathways at different dental schools. There is very little research on efficiency in dental school clinics other than the Kentucky and Harvard studies. ${ }^{1,2}$ When the scope is broadened to consider process automation in dentistry, one study focused on laboratory processes (rather than clinical processes) found that error rates and patient safety improved. ${ }^{6}$ These findings suggest that process automation may improve the overall efficiency of a process.

Traditionally at the University of Michigan School of Dentistry (UMSOD), students needing approval of their treatments have left their cubicles to form lines behind the faculty member they needed. This line of students would follow the faculty member around from patient to patient like following the Pied Piper, with students thus leaving their own patients alone. Patient feedback at the UMSOD showed that, while generally very happy, patients were most concerned about the amount of time waiting during (not before) an appointment. A previous study found that perceived waiting time determined overall patient satisfaction, ${ }^{6}$ so changing this perception could improve satisfaction in our dental school clinic.

UMSOD's information technology unit collaborated with the senior associate dean, associate dean for faculty affairs and institutional effectiveness, and director of predoctoral clinical education to automate the process of requesting faculty by designing and building an electronic Faculty Request System (FRS). Automating this process enabled students to remain with their patients while calling for faculty members electronically. We advised our students to use the additional time with patients to enhance the patient experience through social conversation, detailed discussion about prevention, and even the delivery of additional services.

Automation of emergency services has been found to improve in-hospital and pre-hospital emergency care delivery. ${ }^{7}$ In that study, implementation of the automated system enabled better collaboration and cooperation due to better information exchange. This improvement was also our experience as the process automation began to provide an enormous amount of business intelligence data that enabled us to identify inefficiencies and improve our operations. Dashboards were created using Tableau (interactive data visualization software Business Intelligence software focused on business intelligence analysis) to analyze data exported from the FRS software.

The aim of this study was to assess the effects of this change, including the quality/process improvement interventions that were possible due to information revealed by the FRS. This project was not originally conceived as a research study; rather, it was a quality improvement technology implementation. However, our post-hoc analysis revealed key findings that may be useful to other dental school clinics.

\section{Methods}

This study started out as a quality improvement project, so retrospective approval was gained from the University of Michigan Medical School Committee on Human Research (HUM00116028) to interpret and present the data in a scientific journal. The FRS project was led by the first and last authors, and the software was designed by the UMSOD's own information technology staff.

All data gathered by the FRS were set up to directly populate Tableau in real time. Dashboards were created from all the data and visualized in charts and graphs (led by the second author). Line charts were used to analyze daily, weekly, monthly, and yearly trends. Line histograms were used to analyze peak periods, weekday, request type, and discipline requested. Box plots were used to analyze weekdays and session utilization. Stacked bar graphs were used to track trends in the number of appointments. Scatter charts were used to identify under- and overutilized faculty members. From these graphs, two types of dashboards were created: live and summary. We evaluated business intelligence data from FRS collected between October 2015 and June 2018. The data were generated from requests made by every single student in the second, third, or fourth year during $2015,2016,2017$, and 2018 as there is no other way to make requests for faculty except by using FRS.

The FRS has 39 distinct types of requests distributed across six disciplines: dental assisting, endodontics, oral medicine, periodontics, prosthodontics, and restorative dentistry. This large volume of categorized, time-stamped information is valuable for use as business intelligence data. We are able to track the progress of cases by the type of request (for example, a request to start appointment, request to check a step of appointment, request to finish and check out of appointment). Using this data, we are able to identify bottlenecks, inefficiencies, and 
opportunities for improvement. We identified several issues, implemented change strategies, and continued measuring to evaluate the outcomes.

In addition, we completed an evaluation of how much time the patients were left alone before and after implementation of FRS. This measurement was not the business intelligence part of our study and was very resource-heavy as two evaluators shadowed one student provider for an entire clinic session to measure the amount of time the patient was left alone. In total, 65 student-patient interactions were measured over two weeks (the first was two months before implementation of the FRS, and the second was two months after). Cubicles were randomly selected by putting all cubicle numbers into an online random number generator. Students in those cubicles were selected to be measured.

\section{Results}

It is important to emphasize that there was no increase or decrease in the number of students per clinic session or the number of faculty supervisors per clinic session throughout the three years of this quality improvement evaluation. In fact, the number of procedures completed was quite stable, with small increases from 2015 to 2016 of $1.5 \%$; from 2016 to 2017 of $1.2 \%$; and from 2017 to 2018 of $1.2 \%$. To determine the non-value-added time (i.e., time students spent away from their patients), we measured the amount of time patients were left alone while the student was following the faculty member around before and after the FRS was implemented (Figure 1; FRS was implemented at the $40^{\text {th }}$ measure). The amount of time students spent away from their patients decreased from an average 40.6 minutes to 12.1 minutes $(70.2 \%$ reduction).

The second evaluation considered business intelligence data from FRS that were collected between October 2015 and June 2018. In total, we reviewed 204,445 requests for faculty made through the FRS over that period. This number was for 40,509 unique patient visits. Figure 2 shows the total number of requests for faculty (blue bar) and average number of requests per clinic session by discipline (red line). Restorative dentists had, by far, the most requests at 148,382. However, prosthodontic dentists had the most requests per appointment with an average of 6.75 per visit.

We also calculated the distribution of start requests (the time the appointment starts) before and after quality improvement interventions. After the interventions, there was an important reduction in the proportion of students who started their patient cases 60 minutes after the start of session (from 10.3\% of all start checks to $5.6 \%$ ) (Figure 3). There was also

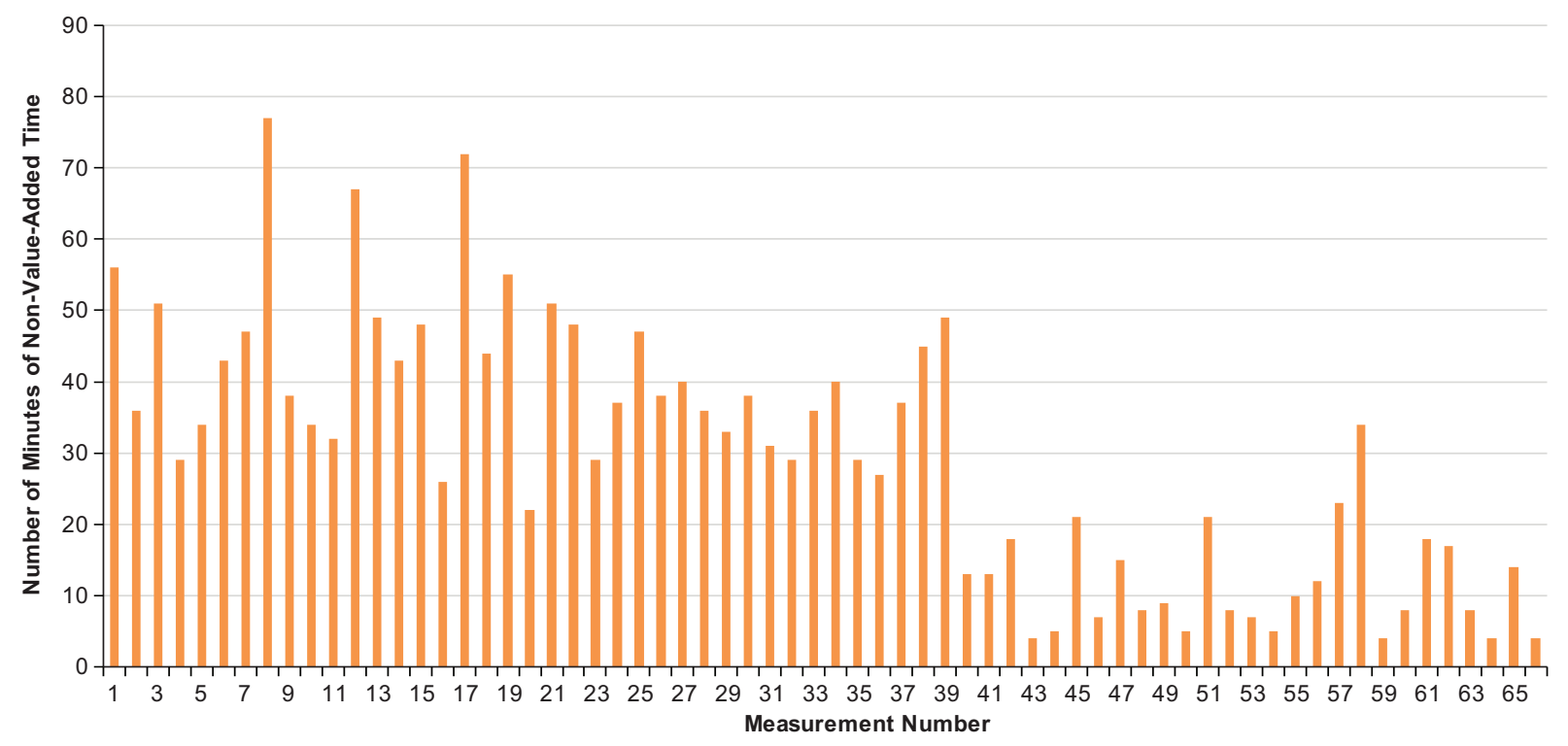

Figure 1. Minutes of non-value-added time, with the Faculty Request System implemented at measurement 40

Note: "Non-value-added time" is time students spent away from their patients. 


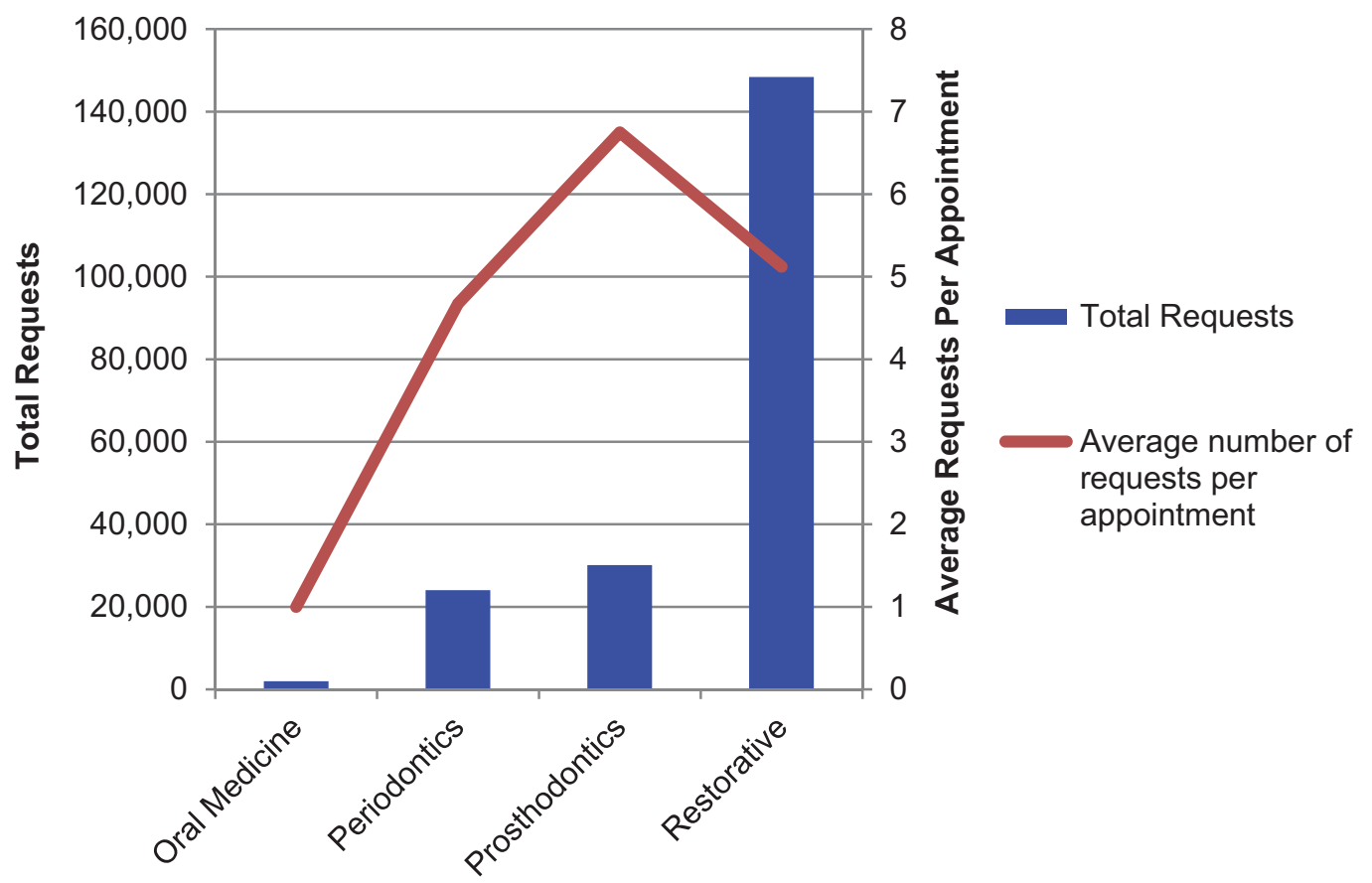

Figure 2. Total number of requests for faculty and average number of requests per appointment by discipline, October 2015 to June 2018

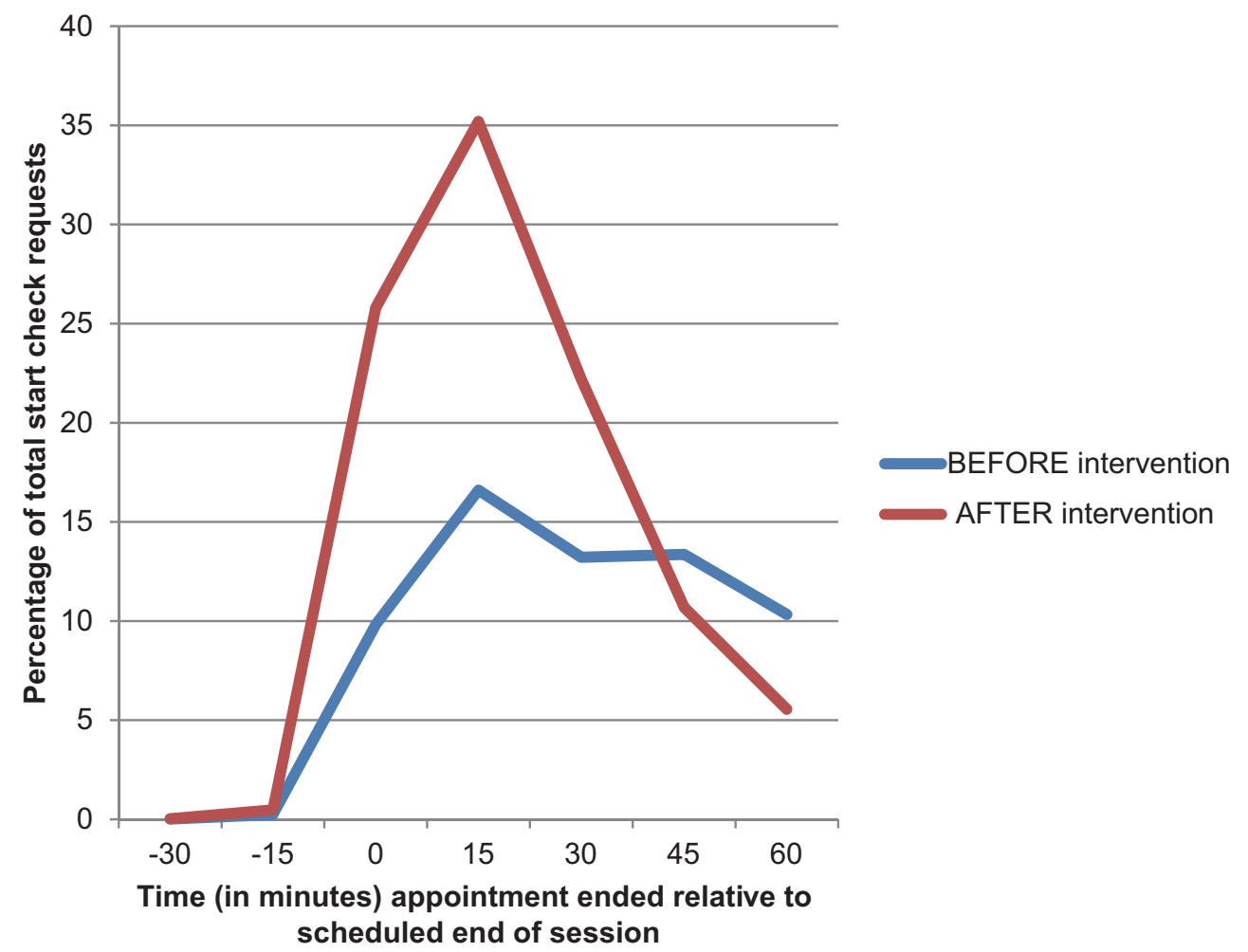

Figure 3. Time of start check requests for faculty made by students: before vs. after intervention 
a small reduction in the proportion of students who started their patient cases 45 minutes after the start of the session (from $13.4 \%$ of all start checks to $10.7 \%$ ). Appointments starting on time showed an improvement after intervention (25.8\% started on time compared to $9.8 \%$ prior to intervention), and $16.6 \%$ were 15 minutes late rather than $35.2 \%$ before intervention. Additionally, only $13.2 \%$ were 30 minutes late compared to $22.2 \%$ prior to intervention. The percentages that started on time $(9.82 \%$ of start checks to $25.8 \%$ ) and 15 minutes (16.6\% to $35.2 \%$ ) and 30 minutes (13.2\% to $22.2 \%)$ after start of session experienced large increases.

Figure 4 shows the distribution of check-out requests (the time the appointment ended) before and after quality improvement interventions. Prior to the interventions, there was only a small percentage of appointments finishing 30 minutes late $(0.03 \%)$; however, after intervention, this practice was completely eradicated. There was also a reduction of appointments ending 15 minutes late (from $0.25 \%$ to $0.01 \%$ ). Interestingly, there was a reduction of appointments finishing at exactly the end time $(1.92 \%$ to $0.4 \%$ ). There was an important increase in the percentage of appointments ending 120, 105, 90, 75, 60 , and 45 minutes early.

Finally, we calculated the mean number of requests per session. After an intervention to educate students about these data and encouraging the use of less busy sessions, there was a slight reduction in number of requests in the busiest sessions (Monday PM went from 223.3 requests to 216.0; Wednesday AM went from 225.5 to 219.0) (Figure 5). There was increased utilization in the slowest session (Friday AM went from 169.9 requests to 193.0).

\section{Discussion}

The FRS was implemented to eradicate the Pied Piper effect and enable students to stay with their patients during the appointment. The valuable business intelligence data were an unexpected bonus to our primary goal. The amount of time students spent away from their patient (non-value-added time) was reduced from an average of 40.6 minutes to $12.1 \mathrm{~min}$ utes $(70.2 \%$ reduction) (Figure 1$)$. This improvement enabled students to have more time for patient-related activity, and we educated them about effective ways to spend this time: getting to know their patient a little more, delivering more preventive services, and taking care of other administrative tasks like scheduling the next appointment and writing the appointment note. However, we acknowledge that this benefit may not have been gained, and students could have used the time for activities unrelated to the patient's care.

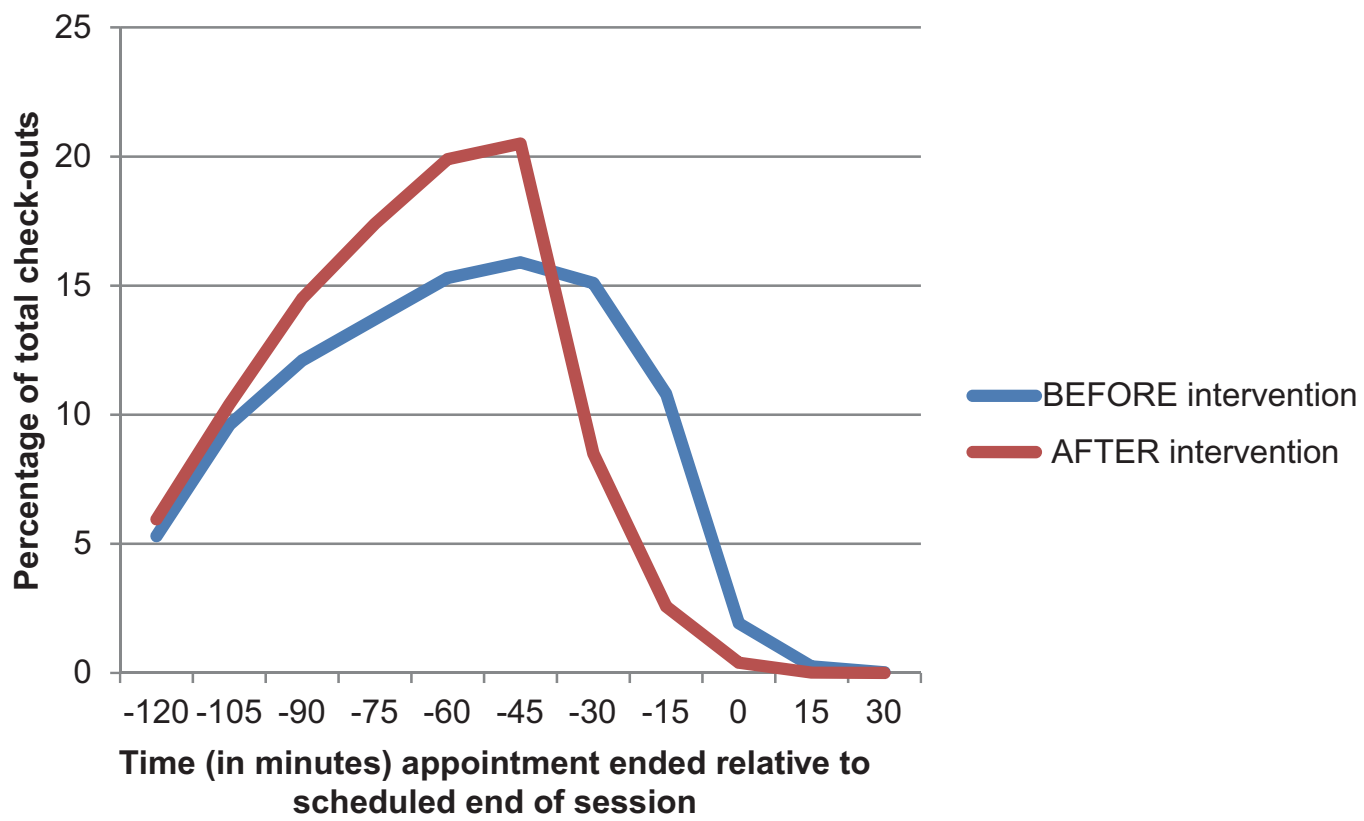

Figure 4. Time of check-out requests for faculty made by students: before vs. after intervention 


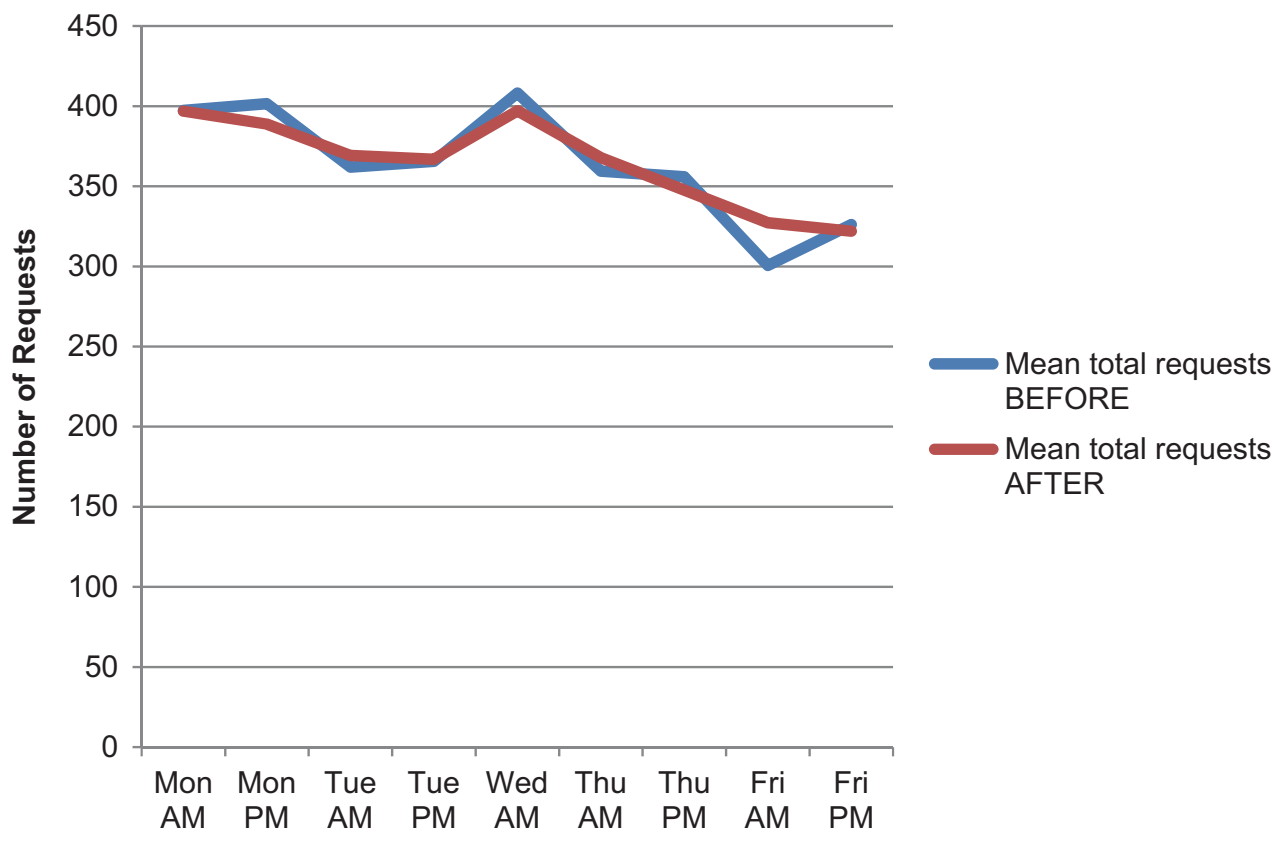

Figure 5. Mean number of requests per session: before vs. after intervention

Note: There is no PM session on Wednesday.

When we determined the average number of requests per appointment and the total number of requests by discipline, restorative dentists (who supervise the largest variety of procedures in our teaching model) clearly had the most requests (Figure 2). It is notable that prosthodontic dentists had the most requests per appointment; this was probably because they are often supervising complex, multistep procedures. Interestingly, we also found a natural variation among students in the number of requests per appointment. We discuss other outcomes under the subsequent headings.

Requests per chair: how to be efficient. The business intelligence data revealed that some students were highly efficient and completed their procedures with only three or four requests for faculty. However, other students required eight to ten requests for the same procedure. When we observed individual "inefficient" students, we realized that a lot of their inefficiency was the way they used the electronic health record. They were requesting faculty members to approve a note, then requesting them to approve the procedure, and requesting them again to approve a referral or complete other paperwork. The most efficient students were batching items to allow faculty to approve multiple items in one visit.
To resolve this inefficiency, we introduced checklists to inform students how to be most efficient each time they requested their faculty members. In this pilot implementation of the checklist, we targeted examination appointments because they occur in high volume and any gains would have large effects. We have been able to reduce faculty requests from a mean of 5.14 to a mean of 3.83 for examinations. Based on mean number of examinations per day, this could reduce the number of requests for faculty by approximately 60 per day.

Requests per faculty: preventing bottlenecks. Currently, our school allows students to choose the faculty member with whom they would like to work. For a variety of reasons, students prefer to work with certain faculty members. Occasionally, a complex case dictates that a student has to keep working with the same faculty member, but sometimes students choose to do so because of a chemistry and rapport that have formed. However, the FRS data revealed that students were choosing specific faculty members despite a detrimental effect on the students' own efficiency because of long wait times.

An example is a morning session on October 7, 2016, when three faculty members-designated Green, Yellow, and Orange-were working with 
12, eight, and four students, respectively. The average wait time for Green on this day was 19 minutes, whereas the average wait time for Orange was four minutes. Recognizing that issues like this were commonplace, we advised faculty members to collaborate and offload additional students to less busy colleagues. We also advised students to choose the least busy faculty members. Although both of these interventions had some effect on reducing the bottleneck for certain instructors, it did not resolve the problem. We have now programmed the FRS software so that a fourth student can only request Green if Yellow and Orange also have three students. We have built the same limitations at six, nine, and 12. This reallocation will reduce the mean wait time for faculty members and completely smooth the variability that used to exist among them.

Thank goodness it's Friday? The UMSOD has nine clinical sessions of equal length (three hours each). This means that each session represents $11.1 \%$ of the week; however, we found that faculty request distribution did not fit this neat pattern. There was a general downward trend in number of requests (and, therefore, busy-ness of the clinics) from Monday to Friday (Figure 5). In fact, the Friday AM session was the least busy session with only $9.17 \%$ of the week's appointments. That session was closely followed by Friday PM with $9.95 \%$ and Thursday PM with $10.9 \%$. In contrast, Wednesday AM (a day when there is no PM session) represented $12.5 \%$ of the week's requests, Monday PM represented 12.3\%, and Monday PM represented 12.1\%.

Through the student clinic representatives, students raised some concerns about perceived faculty shortages during extremely busy clinic sessions when students felt they could not be productive because they were waiting for faculty approvals for so long. Using data from the FRS, we advised students that there were more opportunities on Fridays as there was the same number of faculty members but fewer requests per session. Anecdotally, we heard back from clinic representatives that some students were actively moving patients to Friday appointments. There has been a need to remind students about this pattern every six months as there tended to be a shift back to inefficient Fridays without those reminders. About 18 months after we first advised student providers (through emails, education at clinic orientation, and verbally through student leaders), we evaluated the FRS data again and found that Fridays, which used to represent $19.1 \%$ of requests, had grown slightly to $19.8 \%$. This small percentage change represented a numerical shift of 23 additional requests every Friday across all predoctoral clinics.

Delayed start times. Our business intelligence data from FRS revealed that the mode start time was 15 minutes after the official start of a clinic session. Evaluation of this problem revealed a series of conflicting issues. Lectures are supposed to end ten minutes before a clinic session begins; however, we found that some lectures would continue until two or three minutes before a clinic session, which delays student arrival into clinic. Subsequently, clinical faculty members who noted regular tardiness by students started arriving 15 minutes later to clinic themselves. These delays meant that even if a lecture finished on time, the students could not start clinic on time due to faculty absence.

We worked with our dental assistants and dispensing staff to re-imagine start-up processes at the beginning of clinic sessions. First, we negotiated with students and staff to transfer some responsibilities of chair set-up from students to assistants. Second, instrument dispensing moved from just-in-time to day-before online ordering and day-of pick-ups. Instead of waiting in long lines on the appointment day for their equipment, students could now pre-order online and quickly pick up their equipment with no wait. These more efficient pre-session practices have had an unexpected effect: there are even more students starting 15 minutes after the start of session. However, there has been an important reduction in students who started at 60 and 45 minutes into the session (Figure 3). Overall, we were able to more than double the proportion of "start-checks" that occurred on time at the start of clinic session.

Trends in end times. We evaluated the duration of appointment for all 40,509 appointments. A total of 8,851 (or $21.8 \%$ ) of these had no measurable "end time" because students had failed to use the FRS for the final check. For example, the faculty member may have been walking past at the end of the session, and it would have been more tedious to make an electronic request than to ask the instructor to stop. However, for those appointments that had end time information, $0.03 \%$ ended 30 minutes late, $0.25 \%$ finished 15 minutes late, and $1.92 \%$ finished at the end of session. Over 97\% finished early: 5.3\% finished two hours early, 9.6\% finished 105 minutes early, $12.1 \%$ finished 90 minutes early, $13.7 \%$ finished 75 minutes early, and $15.3 \%$ finished one hour early. This information showed us that many procedures could be completed in less than two hours. With efforts to start on time, we found that even more 
appointments were completed in less than two hours, which informed the next intervention.

Shorter appointments. With the collected data, we discovered trends that we did not know existed but warranted intervention. By tracking the start time and end time in the FRS, we were able to calculate that the mode for duration of appointment in restorative dentistry was two hours 15 minutes $(n=6,138)$ with a second peak at two hours 30 minutes $(n=5,959)$. These data showed that students do not need the entire three hours we offer to complete a restorative appointment. In fact, we calculated that one week included approximately 200 hours of unused chair time across all 144 predoctoral dental chairs. The mode for duration of appointment in prosthodontics was one hour and 45 minutes $(n=969)$, which means that the dental chair was empty in those appointments for one hour and 15 minutes. However, two more peaks were revealed. There was another peak at one hour $(\mathrm{n}=796)$, meaning the dental chair was empty for two hours, and another peak at two hours and 30 minutes $(n=688)$, which was closer to the expected finish time. Periodontal appointments had a mode for duration of two hours and 15 minutes $(n=853)$, and there were no other remarkable peaks. Those data indicated that 45 minutes of additional chair time was available in most periodontal clinic appointments.

A problem that many dental school clinics have is that front desk staff are inundated by patients at the start and end of sessions. These create additional bottlenecks that affect timely start of clinical care delivery and timely departure of patients. With all of this information, we completely re-imagined our traditional appointment times and piloted a morning session that had one-hour, two-hour, and three-hour appointments available. This change had mixed effects because students were hesitant to use the abbreviated start times due to the availability of traditional three-hour slots. Since students realized that our pilot of this change affected only $40 \%$ of dental chairs in one of the nine sessions for one term, they infrequently used these shorter appointment times. Those who used shorter appointments all finished on time, and there was a reduced bottleneck effect on the front desk staff. We plan a broader pilot across two full days throughout all predoctoral clinics, so that students will not have the option to wait for a traditional three-hour appointment time.

Additionally, the business intelligence data revealed that there was a reduction in demand for faculty about 30 minutes after the mode of the start time (which was 15 minutes after start of session). This trend was similar across all disciplines. Therefore, we also piloted staggered appointment times to enable a quarter of students to start later and have their faculty requests occur during that lull period. The pilot was conducted over two terms with 80 sessions (320 appointments). In this time, 262 appointments were kept ( 58 failed) by patients and were evaluated. Of the 262, a total of 91 started 30 minutes later (and were two and a half hours long instead of three hours). Overall, $96.7 \%$ of these staggered and abbreviated appointments finished on time compared to $97.0 \%$ of the regular three-hour appointments. Clearly, it is feasible to have shorter appointments that are thoughtfully staggered to allow more efficient use of faculty time. Moving forward, we will implement shorter appointments that are coupled with staggered start times. This new clinic model has enabled us to move to 412 mixed shorter and longer appointments per day (with staggered start times) from 288 threehour sessions per day (with the same start time) - a $43 \%$ increase in capacity. We have piloted shorter appointments in various forms, and, starting in summer 2019, we will move to the mixed short-and-long appointment model across all five days of the week, which will become our modus operandi.

Mid-term lull and summative clinical assessments. Historically, our school has chosen to have students demonstrate clinical skill progression by requiring them to complete increasingly complex summative assessments as the terms progress through their DDS program. Students are eligible to take these from the first day of term, but every term has a dramatic increase in requests to supervise summative assessments towards the end of term with many days having requests more than three standard deviations above the mean. A major issue is that many summative assessments require two faculty members to supervise, which creates huge bottlenecks at the start and end of term. We noticed that there was a midterm lull in requests for faculty members to supervise summative assessments (Figure 6). Deeper study revealed the lull was caused by two student practices: intense, last-minute stress for less-organized students toward the end of term, and highly organized students' holding back eligible cases to complete a summative exam at the very start of the next term. We confirmed this with case reviews and interviews with students. We realized our assessment system was inadvertently affecting clinical case progression and causing students to moderate care delivery to align with summative assessments. 


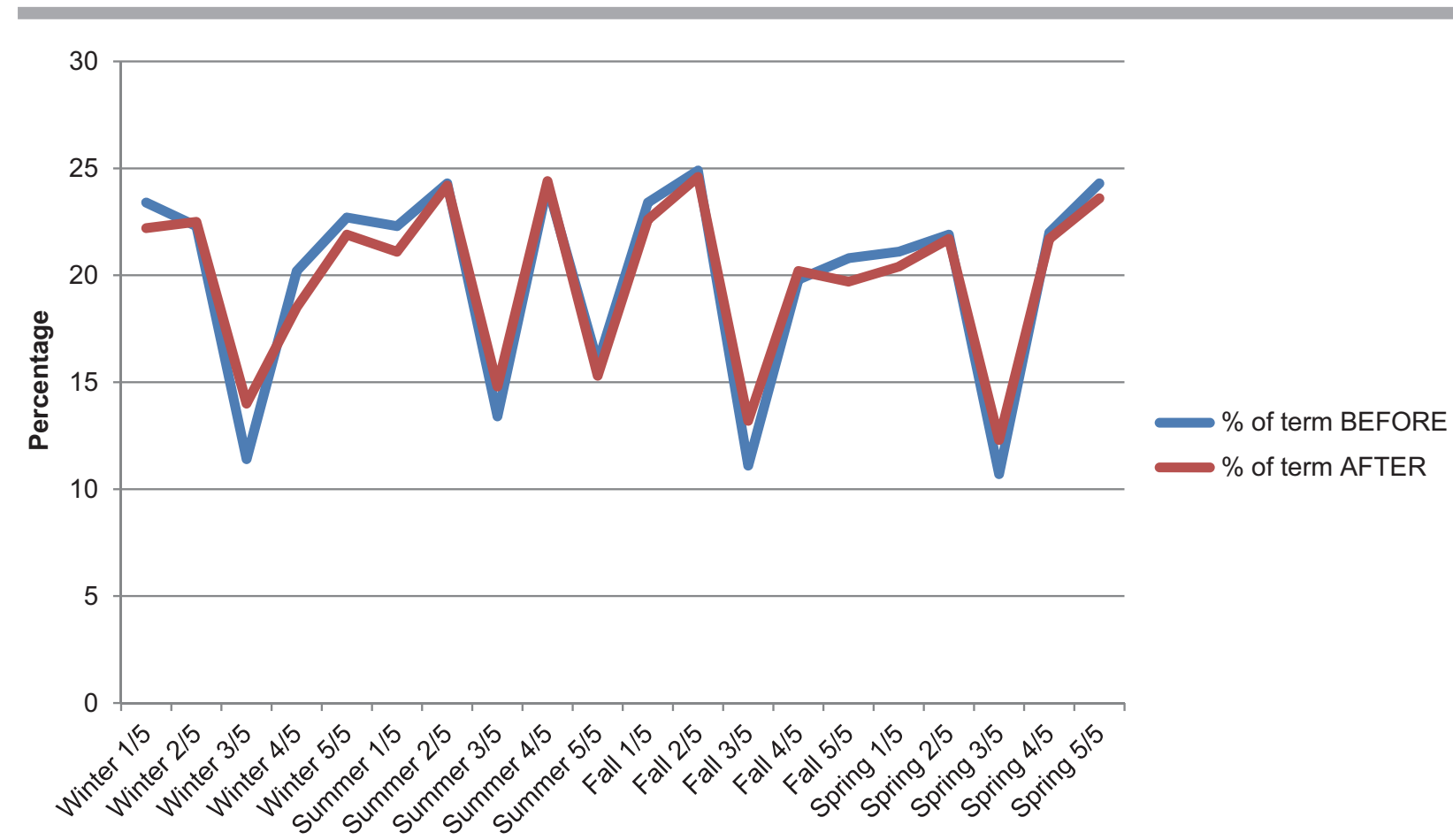

Figure 6. Mid-term lull seen in percentage of summative assessments in each term: before and after intervention

Note: Terms are broken down into five segments, so, for example, "Winter 1/5" label on horizontal axis refers to the first of five segments in the winter term.

Our faculty members were very concerned with this finding (which, although suspected, could not have been confirmed without the FRS data), and we are now moving toward a completely different model of assessment that allows students to have several months to complete a series of summative assessments with progression measured by holistic review. In the interim, we educated students about completing these assessments when eligible cases presented rather than waiting for "ideal" cases and rushing at the end of term. In addition, we implemented new curricular flexibility to allow students to take some summative assessments a term early if they had an eligible case. We have also negotiated with our provost to allow an "incomplete" grade (rather than a fail grade) for students who did not find a suitable case during a certain term. They are able to catch up in the subsequent term with no grade penalty. There has been a small reduction of the mid-term lull in every term, but the largest change was in winter term.

Real-time adaptability. For the first time, with the FRS data a live view of faculty activity across all four predoctoral dental clinics was possible. These real-time data provide support for adjusting faculty distribution mid-session. This change has enabled us to schedule a "floater" who can move to a specific area of need based on the busy-ness of peer faculty.

This study had some limitations. All of these data were collected and evaluated for the purpose of quality improvement and not with the rigor of a study. Procedures, patients, and students were not standardized before and after each intervention, and all results are gained from aggregate data, not from specifically matched pre- and post-evaluations of individuals. However, we believe we have gained valuable data to inform clinical operation decision making by considering the business intelligence data generated from the FRS.

\section{Conclusion}

By critically analyzing data from the Faculty Request System, dental school leadership can analyze trends and make data-driven decisions to alter operations. This process can improve patient care, faculty and facility utilization, and the student experience. We hope that other schools will consider 
using a similar system to make data-driven efficiency improvements. Additionally, our specific discoveries and interventions may be valuable to other dental schools.

\section{Acknowledgments}

This study is based on business intelligence data developed from the University of Michigan's Faculty Request System. That system could not have been built and implemented without tireless support to the authors from Dr. Lynn Johnson, Roger Gillie, Michael Bleed, Dr. Sara Safdari-Sadaloo, and Dr. John Bazzi. We thank you.

\section{REFERENCES}

1. Robinson FG, Cunningham LL, Turner SP, et al. Improving a dental school's clinic operations using lean process improvement. J Dent Educ 2016;80(10):1170-9.
2. Perez FA, Allareddy V, Howell H, Karimbux N. Comparison of clinical productivity of senior dental students in a dental school teaching clinic versus community externship rotations. J Dent Educ 2010;74(10):1125-32.

3. Ten Cate O. Nuts and bolts of entrustable professional activities. J Grad Med Educ 2013;5(1):157-8.

4. Reinhardt JW. Current status of operation and management of dental school clinics. J Dent Educ 2017;81(8 Suppl):eS50-4.

5. Nalliah RP, Forman MS, Chavis SE, Timothé P. Variability between credit units dedicated to dental and clinical sciences in dental schools across the USA. J Investig Clin Dent 2017;8(3).

6. Fontova-Almató A, Juvinyà-Canal D, Suñer-Soler R. Influence of waiting time on patient and companion satisfaction. Rev Calid Asist 2015;30(1):10-6.

7. Seaberg RS, Stallone RO, Statland BE. The role of total laboratory automation in a consolidated laboratory network. Clin Chem 2000;46(5):751-6. 